\title{
Sentinel node navigation surgery attenuates the functional disorders in early gastric cancer
}

\author{
YOSHIHISA YAGUCHI, HIRONORI TSUJIMOTO, ISAO KUMANO, RISA TAKAHATA, \\ YUSUKE MATSUMOTO, KAZUMICHI YOSHIDA, HIROYUKI HORIGUCHI, SATOSHI ONO, \\ TAKASHI ICHIKURA, JUNJI YAMAMOTO and KAZUO HASE
}

Department of Surgery, National Defense Medical College, 3-2 Namiki, Tokorozawa, Saitama 359-8513, Japan

Received September 7, 2011; Accepted October 27, 2011

DOI: 10.3892/or.2011.1568

\begin{abstract}
The purpose of this study was to evaluate the merits of the sentinel node ( $\mathrm{SN}$ )-navigated reduced gastrectomy (SNRG) procedure. The subjects (sT1N0) were divided into the SNRG group $(n=34)$ and the GL group, that consisted of patients which underwent gastrectomy according to the Japanese Gastric Cancer Association guidelines $(n=33)$. We compared the area of the resected stomach and evaluated their body weight changes, and the results of a questionnaire survey about postoperative symptoms, and nutritional effects by blood tests administered at postoperative months (POM) 3,6 and 12. The median area of the resected stomach was $104 \mathrm{~cm}^{2}$ in the SNRG group vs. $192 \mathrm{~cm}^{2}$ in the GL group. The body weight loss ratio was $-5.9 \pm 5.8$ vs. $-9.3 \pm 4.1 \%$ at POM 3 , and the henoglobin $(\mathrm{g} / \mathrm{dl})$ change rate was $-1.1 \pm 7.9$ vs. $-6.4 \pm 6.5 \%$ at POM 12 in the SNRG and GL groups, respectively. There were no significant differences regarding the passage of food, reflux, the incidence of dumping syndrome, digestive and excretory function, or general condition and the satisfaction levels of the patients. In conclusion, SNRG has some advantages over GL in terms of postoperative disorders for at least one year after surgery, and is the recommended choice of a surgical procedure for early gastric cancer.
\end{abstract}

\section{Introduction}

Although a curative resection for gastric cancer is essential for improving the survival rate, such surgical procedures can cause persistent functional disorders and reduce the patient's quality of life. In addition, the pathological analyses of resected specimens have revealed that lymph node involvement is found in less than $20 \%$ of patients, and metastasis to the second tier lymph nodes is especially rare in patients with early gastric cancer in Japan (1-4). Recently, sub-total or total

Correspondence to: Dr Hironori Tsujimoto, Department of Surgery, National Defense Medical College, 3-2 Namiki, Tokorozawa, Saitama 359-8513, Japan

E-mail: tsujihi@ndmc.ac.jp

Key words: gastrectomy, clinical outcome, sentinel nodes, quality of life gastrectomy with a reduced lymphadenectomy, depending on the clinical and surgical findings, has been recommended in the guidelines for treating gastric cancer edited by the Japanese Gastric Cancer Association (JGCA guidelines) (5). However, even such gastrectomies frequently cause postoperative functional disorders such as body weight loss, digestive functional changes, and nutritional disorders $(6,7)$.

Since 2003, we have employed a partial or wedge resection of the stomach with the dissection of sentinel node (SN) stations, where SNs are distributed with negative sentinel node biopsies (8-10). In these studies, SN-navigated reduced gastrectomy (SNRG) was considered to be safe and acceptable, although a long-term follow-up period is mandatory for all patients.

In the current study, we evaluated the clinical benefits with regard to the postoperative functional status after a partial or wedge resection of the stomach with SN-navigated lymphadenectomy during the postoperative period for up to 1 year, and the surgical outcome, including morbidity and mortality.

\section{Subjects and methods}

Since October 2003, we have performed an SN-navigated reduced gastrectomy with dissection of SN stations in patients with T1N0M0 gastric cancer with a tumor diameter of $\leq 4 \mathrm{~cm}$ (10). We excluded patients who met the criteria for endoscopic treatment; those with a tumor with invasion limited to the mucosa, with a diameter of $\leq 2 \mathrm{~cm}$, with a differentiated histology, and without ulceration, were excluded (5). We also excluded patients with a primary tumor that could not be resected with a safe margin preserving the pylorus. Barium radiographs, endoscopy, abdominal ultrasonography, and abdominal computed tomography were performed for preoperative evaluation. We informed eligible patients about the SN-navigated reduced gastrectomy with dissection of SN stations, as an optional procedure. Patients were requested to choose which procedure they wanted to be performed [SNRG or standard gastrectomy with lymphadenectomy following the JGCA guidelines (5)], in cases where the pathologic examination by frozen section revealed no metastasis in SNs. Thirty-seven patients received the SNRG with dissection of SN stations between October, 2003 and July, 2008 (SNRG group). Of these, 3 patients showed metastasis in SNs by frozen section evaluation, and underwent D2 gastrectomy during this period, and were thus excluded from this study. 
Table I. Questionnaire regarding the post-operative functional status including questions about diet, passage and reflux, dumping syndrome, digestive and excretory functions and general health conditions and satisfaction levels.

Questionnaires about diet

1) How much food do you normally eat?

Indicate the percentage of single intake to the preoperative value.

2) How often do you have food on daily basis?

$\begin{array}{llll}\text { a. } 2 \text { times }(+ \text { snack) } & \text { b. } 2-3 \text { times } & \text { c. } 3 \text { times }(+ \text { snack }) & \text { d. } 4 \text { times }(+ \text { snack }) \quad \text { e. } 5 \text { times or more }\end{array}$

Questionnaires about passage and reflux

3) How often do you feel food lying heavily on your stomach or is hard-to-swallow?

4) How often do you experience reflux?

5) How often do you experience vomiting?

a. none b. once a/every other month c. once a/every other week d. 2-3 times a week e. almost every day

Questionnaires about dumping syndrome

6) How often do you have palpitation, sweat, face flush, or bodily hot flush within $30 \mathrm{~min}$ after a meal?

7) How often do you have a cold sweat, dizziness, feel general fatigue, or finger tremor a couple of hours following a meal?

a. none b. once a/every other month c. once a/every other week d. 2-3 times a week e. almost every day

Questionnaires about digestive and excretory functions

8) How often do you feel abdominal distention or flatulence?

9) How often do you have diarrhea?

a. none b. once a/every other month c. once a/every other week d. 2-3 times a week e. almost every day

Questionnaires about general conditions and satisfaction levels

10) How is your general health condition?

a. very good b. good c. no opinion d. bad e. very bad

11) Please indicate your current satisfaction level.

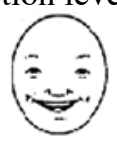

a.

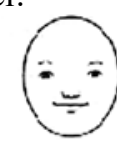

b.

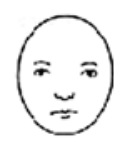

c.

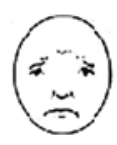

d.

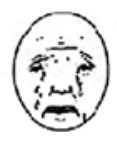

e.
Thirty-three patients served as historical controls from January 2001 to September 2003, who met the inclusion and exclusion criteria for $\mathrm{SN}$-navigated surgery and underwent a standard gastrectomy with lymphadenectomy following the JGCA guidelines (5) (GL group).

We evaluated the area of the resected stomach and performed the questionnaire survey regarding the postoperative gastrointestinal symptoms and nutritional status by blood test up to postoperative month (POM) 12 . This was a nonrandomized and retrospective study.

Surgical procedure. For the SNRG group, we detected the SNs during the surgery by the RI method ( ${ }^{99 \mathrm{~m}} \mathrm{Tc}$-tin colloid) and the Dye method (ICG). When SNs were diagnosed as having negative cancer involvement by examination of intraoperative frozen sections, sleeve or wedge resection of the stomach with lymphadenectomy of the SN stations was performed according to the tumor location (10). All resected specimen were confirmed to be free of cancer cells at the surgical margins by intraoperative frozen section examination.

For the GL group, a total, proximal, distal or pyloruspreserving gastrectomy with a lymph node dissection with D1 lymph node dissection according to the JGCA guidelines was performed, depending on the tumor location (5). D1 lymph node dissection was defined as the removal of perigastric LNs and LNs along the left gastric artery (station \#7) or additional LNs along the common hepatic artery (station \#8a) and/or LNs along the celiac axis (station \#9) according to the depth, size, and the location of the tumor.

Measurement of the area of the resected stomach. A photocopy of the resected stomach, along with a ruler, was scanned and saved as a TIFF file. The area of the resected stomach was determined using the Scion Image for Windows software program (available at: www.scioncorp.com), which is based on the National Institutes of Health ImageJ software for Macintosh developed by Wayne Rasband.

Assessment of postoperative functional status. Questionnaires were administered regarding body weight and postoperative functional status, such as amount of oral intake, passage and reflux, the incidence of dumping syndrome, the digestive and excretory function, and the patient's general condition and satisfaction levels (Table I). Questionnaires were performed at POM 3, 6 and 12. Those time points were chosen based on the usual follow-up schedule after gastrectomy to maximize compliance for the questionnaire and to compare other nutritional parameters at the same time. Nutritional parameters included the serum total cholesterol and albumin levels, total lymphocyte counts, and hemoglobin levels. 
Table II. Demographic data and surgical procedure about the SNRG $(n=34)$ and GL groups $(n=33)$.

\begin{tabular}{|c|c|c|c|}
\hline Characteristics & $\begin{array}{l}\text { SNRG group } \\
\mathrm{n}(\%)^{\mathrm{a}}\end{array}$ & $\begin{array}{l}\text { GL group } \\
\mathrm{n}(\%)^{\mathrm{a}}\end{array}$ & P-value \\
\hline Age (years) & $63 \pm 11$ & $61 \pm 9$ & $\mathrm{P}=0.37$ \\
\hline Gender (male/female) & $24 / 10$ & $22 / 11$ & $\mathrm{P}=0.73$ \\
\hline \multicolumn{4}{|l|}{ Tumor depth } \\
\hline M & $19(55.9)$ & $16(48.5)$ & \multirow[t]{2}{*}{$\mathrm{P}=0.37$} \\
\hline SM & $15(44.1)$ & $17(51.5)$ & \\
\hline \multicolumn{3}{|l|}{ Histological type } & \multirow[t]{3}{*}{$\mathrm{P}=0.92$} \\
\hline Diffuse type & $13(38.2)$ & $13(39.4)$ & \\
\hline Intestinal type & $21(61.8)$ & $20(60.6)$ & \\
\hline Tumor size (mean \pm SD mm) & $29 \pm 15$ & $28 \pm 13$ & $\mathrm{P}=0.75$ \\
\hline \multicolumn{4}{|l|}{ Tumor location } \\
\hline $\mathrm{U}$ & $5(14.7)$ & $8(24.2)$ & \multirow[t]{3}{*}{$\mathrm{P}=0.61$} \\
\hline M & $22(64.7)$ & $19(57.6)$ & \\
\hline $\mathrm{L}$ & $7(20.6)$ & $6(18.2)$ & \\
\hline \multicolumn{3}{|l|}{ Surgical procedure } & \multirow[t]{7}{*}{$\mathrm{P}<0.001$} \\
\hline Total gastrectomy & 0 & 2 & \\
\hline Proximal gastrectomy & 0 & 8 & \\
\hline Distal gastrectomy & 0 & 12 & \\
\hline Pylorus preserving gastrectomy & 0 & 11 & \\
\hline Partial gastrectomy & 9 & 0 & \\
\hline Sleeve gastrectomy & 25 & 0 & \\
\hline Duration of surgery (mean $\pm \mathrm{SD}$ ), min & $211 \pm 36$ & $262 \pm 49$ & $\mathrm{P}<0.01$ \\
\hline Estimated blood loss (median, range), ml & $219,20-1753$ & $309,57-553$ & $\mathrm{P}<0.05$ \\
\hline Hospital stay (median, range), days & $13,8-21$ & $18,14-31$ & $\mathrm{P}<0.01$ \\
\hline Time to liquid diet (median, range), days & $5,3-8$ & $7,5-10$ & $\mathrm{P}<0.01$ \\
\hline Time to the first flatus (median, range), days & $3,2-5$ & $4,2-7$ & $\mathrm{P}=0.14$ \\
\hline Postoperative complications & $4(11.8 \%)$ & $4(12.1 \%)$ & $\mathrm{P}=0.74$ \\
\hline
\end{tabular}

${ }^{\text {a}}$ Values are n (\%) unless otherwise indicated. SD, standard deviation; SNRG, sentinel node (SN)-navigated reduced gastrectomy; GL, group that underwent gastrectomy according to the JGCA guidelines; M, mucosa; SM, submucosa; U, upper third of stomach; M, middle third of stomach; L, lower third of stomach.

Informed consent. The reduced surgery guided by SN biopsy reported in this study was reviewed and approved by the Institutional Review Board at the National Defense Medical College. Written informed consent was obtained from every patient before the procedures.

Statistical analyses. Data are expressed as the mean \pm standard deviation and the median (range). The Mann-Whitney U test, Student's t-test, and the Chi-square test were used for comparisons between the two groups. A P-value $<0.05$ was considered to be statistically significant.

\section{Results}

Demographic data and surgical procedures. There was no significant difference in age, gender, tumor depth, histological type, tumor size, or tumor location between the two groups
(Table II). In the SNRG group, 9 patients underwent a partial gastrectomy and 25 patients underwent a sleeve gastrectomy with reduced lymphadenectomy including the SN station, while in the GL group 2 patients underwent a total gastrectomy, 8 patients underwent a proximal gastrectomy, 11 patients received a pylorus-preserving gastrectomy, and 12 patients underwent a distal gastrectomy with lymphadenectomy according to the JGCA guideline. The duration of the operation, estimated blood loss, postoperative hospital stay, and days until first post-operative oral intake in the SNRG group were significantly better than those in the GL group. With regard to postoperative complications, there were 2 cases of arrhythmia, 1 of gastric stasis, and 1 of anastomotic bleeding in the SNRG group, while there was 1 case each of arrhythmia, gastric stasis, anastomotic leakage, pancreatic fistula, and of wound infection (same case as the pancreatic fistula) in the GL group; there were no significant differences in the postoperative complications. 


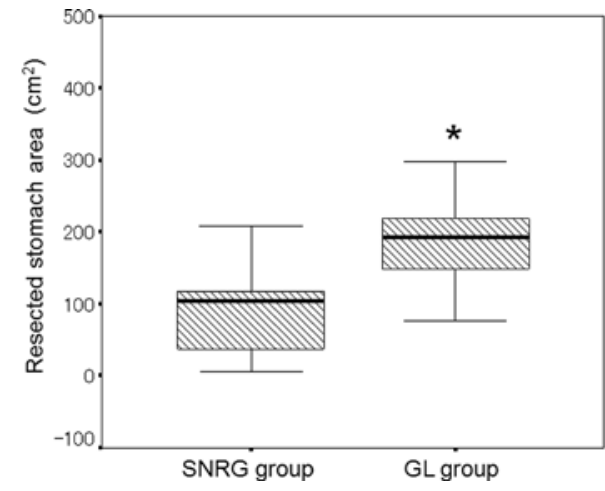

Figure 1. Measurement of the area of the resected stomach using Scion image. The median area of the resected stomach in the SNRG group $\left(104 \mathrm{~cm}^{2}\right.$, range $\left.6-208 \mathrm{~cm}^{2}\right)$ was significantly smaller than that in the GL group $\left(192 \mathrm{~cm}^{2}\right.$, range $75-454 \mathrm{~cm}^{2}$ ). ${ }^{*} \mathrm{P}<0.01$ vs. the $\mathrm{SNRG}$ group.

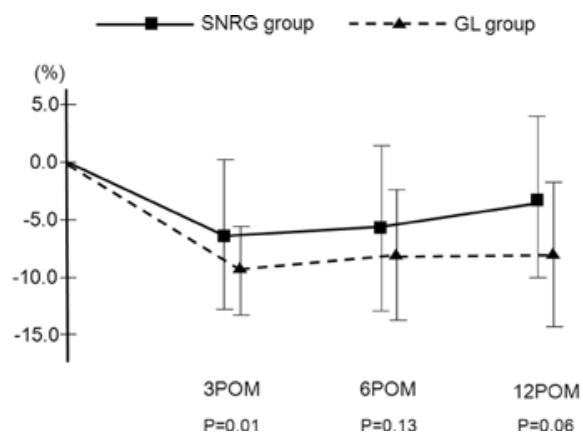

Figure 2. Body weight rate change. The weight loss ratio to the preoperative value (SNRG group vs. the GL group) was $-5.9 \pm 5.8$ vs. $-9.3 \pm 4.1 \%,-5.5 \pm 6.4$ vs. $-8.1 \pm 6.1 \%$ and $-3.9 \pm 7.5$ vs. $-7.9 \pm 6.0 \%$ at POM 3,6 and 12 , respectively. There were significant differences between the two groups at POM $3(\mathrm{P}=0.01)$.

None of the patients had developed recurrence or metastasis, or had died up until the time this manuscript was written.

Area of the resected stomach. We compared the area of the resected stomach between the two groups. The median area of the resected stomach in the SNRG group $\left(104 \mathrm{~cm}^{2}\right.$, range $\left.6-208 \mathrm{~cm}^{2}\right)$ was significantly smaller than that in the GL group $\left(192 \mathrm{~cm}^{2}\right.$, range $75-454 \mathrm{~cm}^{2}$ ) (Fig. 1).

Body weight change. The weight loss ratio to the preoperative value (SNRG group vs. GL group) was $-5.9 \pm 5.8$ vs. $-9.3 \pm 4.1 \%$, $-5.5 \pm 6.4$ vs. $-8.1 \pm 6.1 \%$ and $-3.9 \pm 7.5$ vs. $-7.9 \pm 6.0 \%$ at POM 3 , 6 and 12 , respectively. There were significant differences between the two groups at POM 3 ( $\mathrm{P}=0.01)$ (Fig. 2).

Postoperative functional status. The percentage of the amount of the single oral intake to the preoperative value (SNRG group vs. GL group) was $74 \pm 18$ vs. $66 \pm 17 \%, 76 \pm 16$ vs. $70 \pm 14 \%$ and $81 \pm 16$ vs. $74 \pm 12 \%$ at POM 3,6 and 12 , respectively. There was no significant difference between the two groups (Fig. 3A). There was, however, a significant difference in the frequency of the intake of meals per day between the two groups at POM 6 (Fig. 3B). There were no significant differences regarding the passage of food, reflux, the incidence of dumping syndrome, digestive and excretory function, or general condition and the satisfaction levels of the patients (Figs. 4-7).

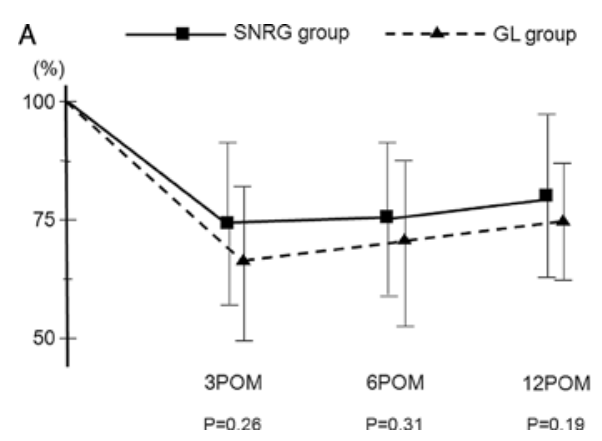

B

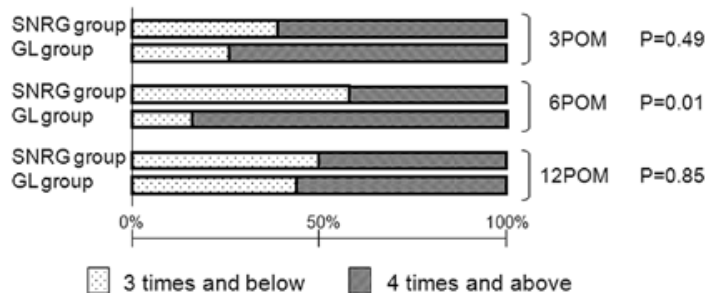

Figure 3. (A) Percentage of single intake to the preoperative value. The percentage of the amount of the single oral intake to the preoperative value (SNRG group vs. the GL group) was $74 \pm 18$ vs. $66 \pm 17 \%$, $76 \pm 16$ vs. $70 \pm 14 \%$ and $81 \pm 16$ vs. $74 \pm 12 \%$ at POM 3,6 and 12 , respectively. There was no significant difference between the two groups. (B) Frequency of the intake of the meals per day. The percentage of intake times per day separated between 3 times and below, and 4 times and above (SNRG group vs. the GL group) was 42 and $58 \%$ vs. 29 and $71 \%, 56$ and $44 \%$ vs. 17 and $83 \%$, and 50 and $50 \%$ vs 47 and 53\% at POM 3, 6 and 12, respectively. There was a significant difference between the two groups at POM $6(\mathrm{P}=0.01)$.

1) How often do you feel food lying heavily on your stomach or is hard-to-swallow?

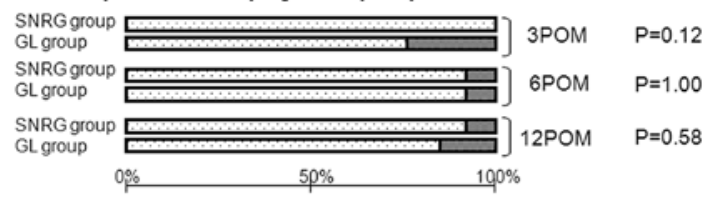

2) How often do you experience reflux?

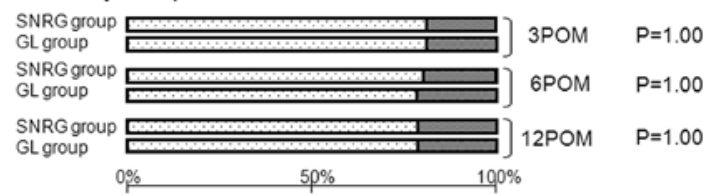

3) How often do you experience vomiting?

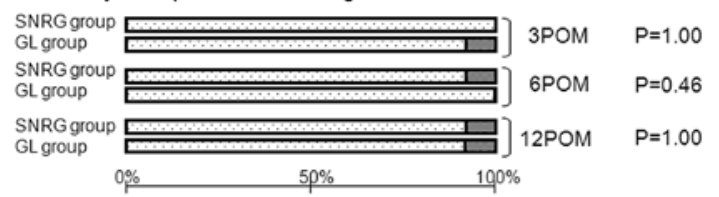

once a/every other month and below $\square$ once a/every other week and above

Figure 4. Questionnaires about passage and reflux. The percentages of patients feeling food lying heavily on their stomachs or is hard-to-swallow separated between once a/every other month and below, and once a/every other week and above (SNRG group vs. the GL group) were 100 and $0 \%$ vs. 76 and 24\%, 93 and $7 \%$ vs. 94 and 6\%, and 94 and $6 \%$ vs. 86 and $14 \%$ at POM 3, 6 and 12, respectively. There was no significant difference between the two groups. The percentage reflux separated between once a/every other month and below, and once a/every other week and above (SNRG group vs. the GL group) was 83 and $17 \%$ vs. 82 and $18 \%, 80$ and $20 \%$ vs. 78 and $22 \%$, and 78 and $22 \%$ vs. 79 and $21 \%$ at POM 3, 6 and 12, respectively. There was no significant difference between the two groups. The percentage of vomiting separated between once a/every other month and below, and once a/every other week and above (SNRG group vs. GL group) was 100 and $0 \%$ vs. 94 and $6 \%, 93$ and $7 \%$ vs. 100 and $0 \%$, and 94 and $6 \%$ vs. 93 and $7 \%$ at POM 3,6 and 12 , respectively. There was no significant difference between the two groups. 
1) How often do you have a palpitation, sweat, face flush, or bodily hot flush within $30 \mathrm{~min}$ after a meal?

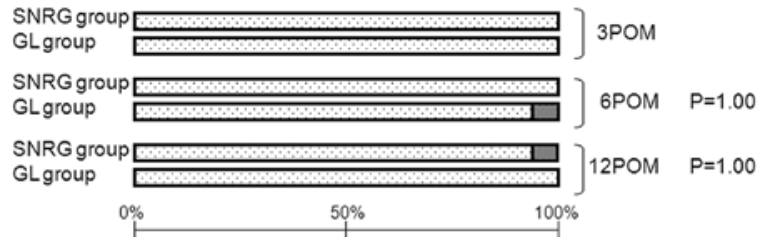

2) How often do you have a cold sweat, dizziness, feel general fatigue, or finger tremor a couple of hours following a meal?

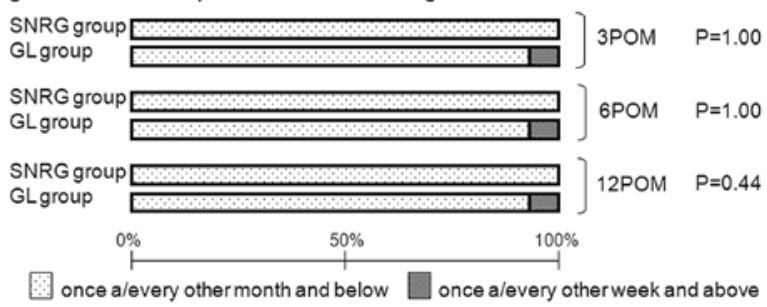

Figure 5. Questionnaires about the dumping syndrome. The percentage of early dumping syndrome separated between once a/every other month and below, and once a/every other week and above (SNRG group vs. the GL group) was 100 and $0 \%$ vs. 100 and $0 \%, 100$ and $0 \%$ vs. 94 and $6 \%$, and 94 and $6 \%$ vs. 100 and $0 \%$ at POM 3, 6 and 12, respectively. There was no significant difference between the two groups. The percentage of late dumping syndrome separated between once a/every other month and below, and once a/every other week and above (SNRG group vs. the GL group) was 100 and $0 \%$ vs. 94 and 6\%, 100 and $0 \%$ vs. 94 and $6 \%$, and 100 and $0 \%$ vs. 93 and $7 \%$ at POM 3,6 and 12 , respectively. There was no significant difference between the two groups.

1) How often do you feel abdominal distention or flatulence?

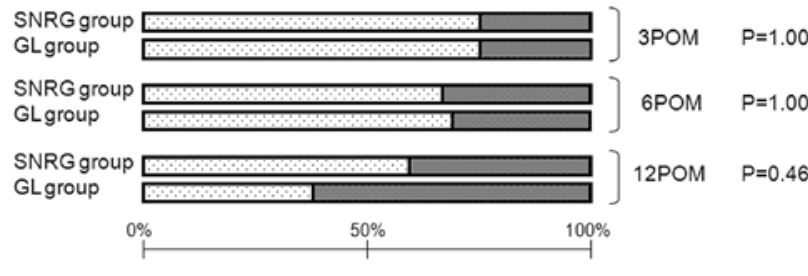

2) How often do you have diarrhea?

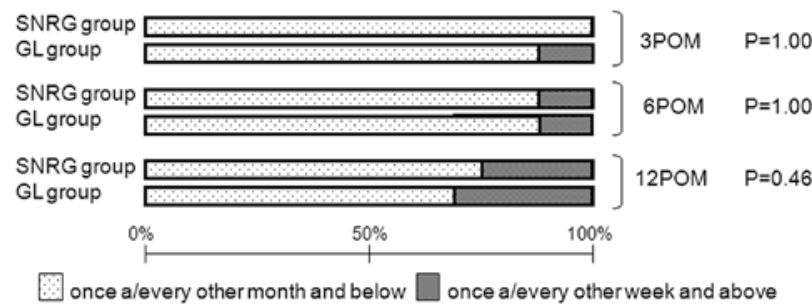

Figure 6. Questionnaires about digestive and excretory function. The percentage of feeling about abdominal distention or flatulence separated between once a/every other month and below, and once a/every other week and above (SNRG group vs. the GL group) was 75 and $25 \%$ vs. 75 and $25 \%$, 67 and $33 \%$ vs. 69 and $31 \%$, and 59 and $41 \%$ vs. 38 and $62 \%$ at POM 3, 6 and 12, respectively. There was no significant difference between the two groups. The percentage of diarrhea separated between once a/every other month and below, and once a/every other week and above (SNRG group vs. the GL group) was 100 and $0 \%$ vs. 88 and $12 \%, 87$ and $13 \%$ vs. 87 and $13 \%$, and 75 and $25 \%$ vs. 69 and 31\% at POM 3, 6 and 12, respectively. There was no significant difference between the two groups.

Nutritional evaluation by blood test. No significant differences were observed in total serum cholesterol and albumin levels, or in the total lymphocyte counts between the two groups
1) How is your general health condition?

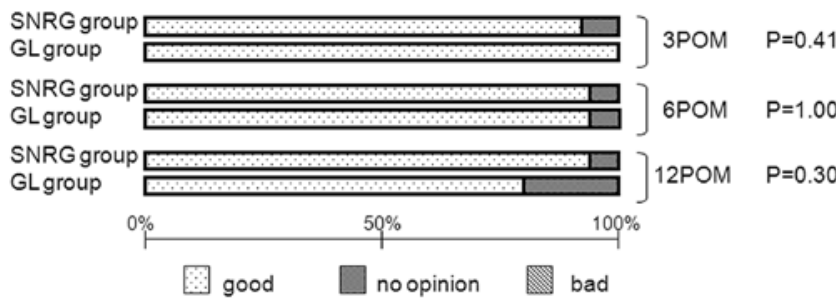

2) Indicate your current overall satisfaction level.

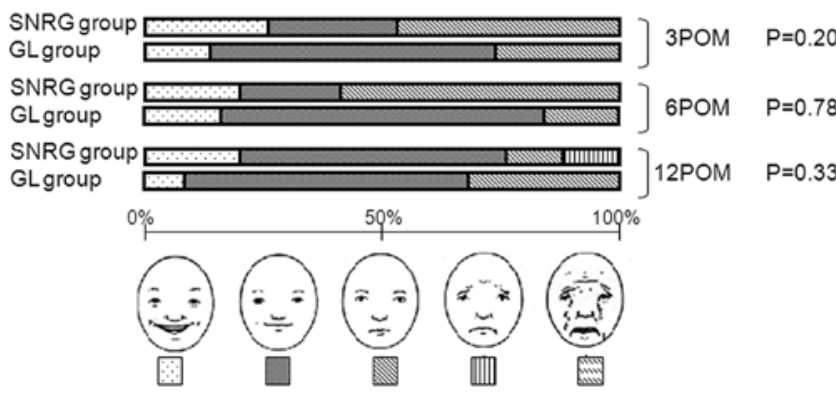

Figure 7. Questionnaires about general condition and satisfaction level. The percentage of general health condition separated good, no opinion and bad (SNRG group vs. the GL group) was 92,8 and $0 \%$ vs. 100,0 and $0 \%, 93,7$ and $0 \%$ vs. 94,6 and $0 \%$, and 94,6 and $0 \%$ vs. 79,21 and $0 \%$ at POM 3,6 and 12 , respectively. There was no significant difference between the two groups. The percentage of overall satisfaction level separated very good, good, no opinion, bad and very bad (SNRG group vs. the GL group) was 27, 27, 46, 0 and $0 \%$ vs. $13,62,25,0$ and $0 \%, 20,20,60,0$ and $0 \%$ vs. $17,66,17,0$ and $0 \%$, and $20,54,13,13$ and $0 \%$ vs. $8,59,33,0$ and $0 \%$ at POM 3,6 and 12 , respectively. There was no significant difference between the two groups.

(Fig. 8A-C). The change in hemoglobin levels were $-5.1 \pm 6.8$ vs $-5.6 \pm 4.3 \%,-2.8 \pm 7.0$ vs. $-6.0 \pm 8.0 \%$ and $-1.1 \pm 7.9$ vs. $-6.4 \pm 6.5 \%$, at POM 3, 6 and 12, respectively. There was a significant difference between two groups at POM $12(\mathrm{P}=0.02)$ (Fig. 8D).

\section{Discussion}

In the present study, we demonstrated that the reduced gastrectomy with SN navigation was superior to the conventional gastrectomy on the basis of the JGCA guidelines in terms of length of postoperative stay, days until postoperative first oral intake, and short-term outcome, such as postoperative weight loss and hemoglobin levels, for early gastric cancer patients.

Malnutrition after gastrectomy is frequently serious, and body weight loss is one of the most common indicators of this problem (11-13). This is especially true of patients who undergo a total gastrectomy, because they have a more dramatic loss of body weight than those who undergo a subtotal gastrectomy $(11,12)$, and the recovery from body weight loss often requires several years $(11,12)$.

Shibata et al (7) reported that the postoperative decrease in body weight at a mean of over 3 years was more than $6 \mathrm{~kg}$ both in the PPG and the conventional distal gastrectomy groups (7). In addition, Takahashi et al (14) reported that the wedge resection of the stomach for early gastric cancer was superior to standard distal gastrectomy in terms of changes in body weight and body fat mass. They found that the change in the volume of postoperative food eaten returned to close to the preoperative levels within a short period, and this observation suggested that the residual volume of the stomach affected the incidence 
A

Total serum cholesterol rate change ( $\mathrm{mg} / \mathrm{dl})$

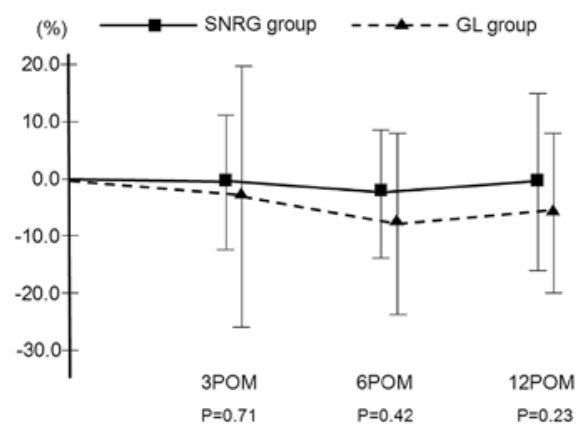

C Lymphocyte count rate change $\left(\times 10^{3 / \mu l}\right)$

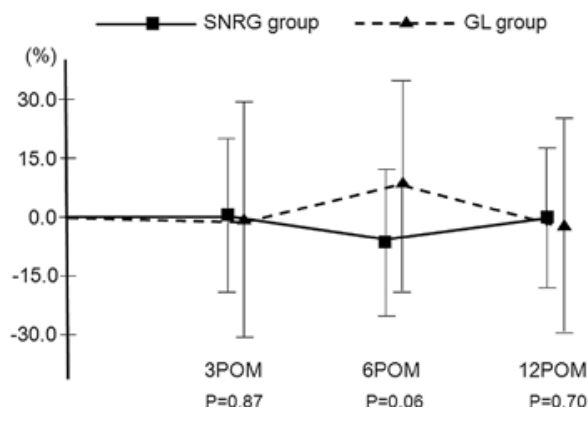

Serum albumin rate change $(\mathrm{g} / \mathrm{dl})$

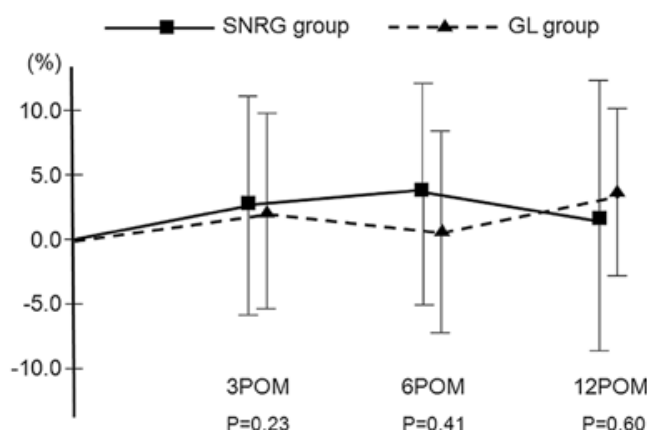

Hemoglobin rate change $(\mathrm{g} / \mathrm{dl})$

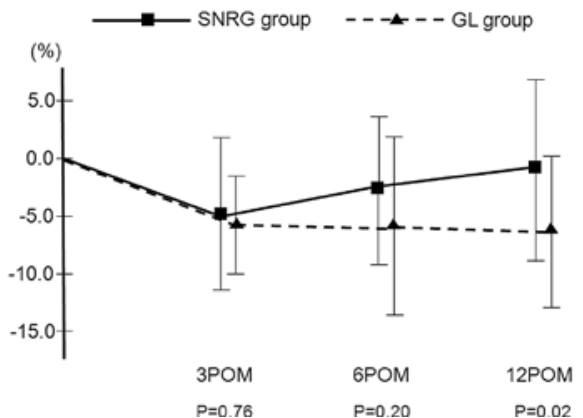

Figure 8. (A) Nutritional evaluation by blood test. Total serum cholesterol rate change. The change of serum total cholesterol levels (SNLG vs. the GL group) were $-0.6 \pm 12.1$ vs. $-3.4 \pm 23.3 \%,-2.4 \pm 11.6$ vs. $-7.6 \pm 15.6 \%$ and $-0.4 \pm 13.4$ vs. $-6.2 \pm 14.3 \%$, at POM 3,6 and 12 , respectively. There was no significant difference between the two groups. (B) Serum albumin rate change. The change of serum albumin levels were $2.6 \pm 8.6$ vs. $2.1 \pm 7.4 \%, 3.6 \pm 8.7$ vs. $0.8 \pm 7.6 \%$ and $1.9 \pm 9.8$ vs. $3.5 \pm 6.7 \%$, at POM 3,6 and 12, respectively. There was no significant difference between the two groups. (C) Lymphocyte count rate change. The change of lymphocytes count were $-0.3 \pm 20.6$ vs. $-1.0 \pm 30.0 \%,-6.6 \pm 19.0$ vs. $7.9 \pm 27.4 \%$ and $-0.4 \pm 17.9$ vs. $-2.8 \pm 25.4 \%$, at POM 3,6 and 12 , respectively There was no significant difference between the two groups. (D) Hemoglobin rate change. The change of hemoglobin levels were $-5.1 \pm 6.8$ vs. $-5.6 \pm 4.3 \%,-2.8 \pm 7.0$ vs. $-6.0 \pm 8.0 \%$ and $-1.1 \pm 7.9$ vs. $-6.4 \pm 6.5 \%$, at POM 3, 6 and 12, respectively. There was a significant difference between the two groups at POM 12 ( $\mathrm{P}=0.02)$.

of postcibal syndromes. In this study, we demonstrated that the resected stomach area of the SNRG group was significantly smaller than that of the GL group, and the body weight rate change in the SNRG group was significantly milder than that in the GL group from early postoperative period. Taken together, these findings indicate that the size of the residual stomach was an important indicator of nutritional status.

There were no significant differences between the two groups in the percentage of single meal intake and the frequency of daily food intake except for POM 6 based on the questionnaire result (Fig. 3). However, the body weight rate change showed a positive correlation with the percentage of single intake $\left(\mathrm{r}^{2}=0.34, \mathrm{P}<0.01\right)$ and negative correlation with the frequency of daily food $\left(\mathrm{r}^{2}=0.19, \mathrm{P}<0.05\right)$ at POM 3 .

We were unable to find an advantage for SNRG with regard to the passage of food, reflux, dumping syndrome, digestive and excretory functions, or the general condition and satisfaction level from the questionnaire survey. Both groups showed acceptable levels according to each symptom, thus an additional accumulation of cases and long-term observation are required to determine whether there is any significant benefit.

The reasons for postoperative anemia were explained by the fact that the decreased acid secretion in the remaining stomach caused a disorder of iron absorption in the upper small intestine. However, the blood tests did not always show an asiderotic anemia pattern. It must be considered that hemoglobin changes are one of the nutritional disturbances that occur with a deterioration in protein synthesis capability, although other blood data such as total serum cholesterol and albumin levels were not significantly different between the two groups. Ryu et al (15) reported that body weight, body mass index, triceps skin fold and midarm circumference were significantly reduced, but the total lymphocyte count, albumin, protein, cholesterol and serum iron levels did not decrease during the postoperative period (at POM 6 and 12) among gastric cancer patients, which was consistent with our results.

In conclusion, a reduced gastrectomy with $\mathrm{SN}$-navigation has several advantages over conventional gastrectomy according to the current guideline in terms of postoperative functional disorders for at least 1 year after surgery, as well as positive impact on the length of postoperative hospital stay, and days until first postoperative oral intake, and it is therefore recommended that this procedure should be the standard treatment for early gastric cancer.

\section{References}

1. Koga S, Kaibara N, Tamura H, Nishidoi H and Kimura O: Cause of late postoperative death in patients with early gastric cancer with special reference to recurrence and the incidence of metachronous primary cancer in other organs. Surgery 96: 511-516, 1984.

2. Holscher AH, Drebber U, Monig SP, Schulte C, Vallbohmer D and Bollschweiler E: Early gastric cancer: lymph node metastasis starts with deep mucosal infiltration. Ann Surg 250: 791-797, 2009. 
3. An JY, Baik YH, Choi MG, Noh JH, Sohn TS and Kim S Predictive factors for lymph node metastasis in early gastric cancer with submucosal invasion: analysis of a single institutional experience. Ann Surg 246: 749-753, 2007.

4. Kunisaki C, Takahashi M, Nagahori Y, et al: Risk factors for lymph node metastasis in histologically poorly differentiated type early gastric cancer. Endoscopy 41: 498-503, 2009.

5. Nakajima T: Gastric cancer treatment guidelines in Japan. Gastric Cancer 5: 1-5, 2002.

6. Nunobe S, Sasako M, Saka M, Fukagawa T, Katai H and Sano T: Symptom evaluation of long-term postoperative outcomes after pylorus-preserving gastrectomy for early gastric cancer. Gastric Cancer 10: 167-172, 2007

7. Shibata C, Shiiba KI, Funayama Y, et al: Outcomes after pyloruspreserving gastrectomy for early gastric cancer: a prospective multicenter trial. World J Surg 28: 857-861, 2004.

8. Ichikura T, Chochi K, Sugasawa H, et al: Individualized surgery for early gastric cancer guided by sentinel node biopsy. Surgery 139: 501-507, 2006

9. Ichikura T, Morita D, Uchida T, et al: Sentinel node concept in gastric carcinoma. World J Surg 26: 318-322, 2002.
10. Ichikura T, Sugasawa H, Sakamoto N, Yaguchi Y, Tsujimoto H and Ono S: Limited gastrectomy with dissection of sentinel node stations for early gastric cancer with negative sentinel node biopsy. Ann Surg 249: 942-947, 2009.

11. Wu CW, Hsieh MC, Lo SS, Lui WY and P'eng FK: Quality of life of patients with gastric adenocarcinoma after curative gastrectomy. World J Surg 21: 777-782, 1997.

12. Kiyama T, Mizutani T, Okuda T, et al: Postoperative changes in body composition after gastrectomy. J Gastrointest Surg 9: 313-319, 2005.

13. Katsube T, Konnno S, Murayama M, et al: Changes of nutritional status after distal gastrectomy in patients with gastric cancer. Hepatogastroenterology 55: 1864-1867, 2008.

14. Takahashi S, Maeta M, Mizusawa K, et al: Long-term postoperative analysis of nutritional status after limited gastrectomy for early gastric cancer. Hepatogastroenterology 45: 889-894, 1998.

15. Ryu KW, Lee JH, Kim HS, Kim YW, Choi IJ and Bae JM Prediction of lymph nodes metastasis by sentinel node biopsy in gastric cancer. Eur J Surg Oncol 29: 895-899, 2003. 